\title{
Noncoding RNA-related polymorphisms in pediatric acute lymphoblastic leukemia susceptibility
}

\author{
Angela Gutierrez-Camino', Elixabet Lopez-Lopez'1, Idoia Martin-Guerrero', Maria A. Piñan², Purificacion Garcia-Miguel',
} Jose Sanchez-Toledo ${ }^{4}$, Ana Carbone Bañeres ${ }^{5}$, Javier Uriz ${ }^{6}$, Aurora Navajas ${ }^{7}$ and Africa Garcia-Orad'

\begin{abstract}
BACKGROUND: Evidence for an inherited genetic risk for pediatric acute lymphoblastic leukemia has been provided in several studies. Most of them focused on coding regions. However, those regions represent only $1.5 \%$ of the entire genome. In acute lymphoblastic leukemia (ALL), it has been suggested that the expression of microRNAs (miRNAs) is dysregulated, which suggests that they may have a role in ALL risk. Changes in miRNA function may occur through single-nucleotide polymorphisms (SNPs). Therefore, the aim of this study was to evaluate whether polymorphisms in premiRNAs, and/or miRNA-processing genes, contribute to a predisposition for childhood ALL.
\end{abstract}

METHODS: In this study, we analyzed 118 SNPs in pre-miRNAs and miRNA-processing genes in 213 B-cell ALL patients and 387 controls.

RESULTS: We found 11 SNPs significantly associated with ALL susceptibility. These included three SNPs present in miRNA genes (miR-612, miR-499, and miR-449b) and eight SNPs present in six miRNA biogenesis pathway genes (TNRC6B, DROSHA, DGCR8, EIF2C1, CNOT1, and CNOT6). Among the 118 SNPs analyzed, rs12803915 in mir-612 and rs3746444 in mir-499 exhibited a more significant association, with a $P$ value $<0.01$

CONCLUSION: The results of this study indicate that SNP rs12803915 located in pre-mir-612, and SNP rs3746444 located in pre-mir-499, may represent novel markers of B-cell ALL susceptibility.

A cute lymphoblastic leukemia (ALL) is the most common pediatric hematological malignancy in developed countries. Its etiology is believed to be multifactorial, with both environmental and genetic risk factors being relevant (1). Recently, several studies have provided evidence for an inherited genetic risk for pediatric ALL $(2,3)$. Most of these studies focused on the coding regions of these genetic components. However, this represents only $\sim 1.5 \%$ of the entire genome, and noncoding regions of the genome have also been shown to mediate regulatory functions. For example, microRNAs (miRNAs) are a class of small noncoding RNA molecules that regulate gene expression at the posttranscriptional level by binding to the $3^{\prime}$ untranslated region of a target gene (4). This can lead to an inhibition of translation or enhanced degradation of a target mRNA (Figure 1). Primary double-stranded miRNA transcripts (pri-miRNA) are processed in the nucleus by microprocessor machinery, which includes DROSHA RNase and the double-stranded RNA-binding protein, DGCR8. A hairpin precursor miRNA molecule of $70-100$ nucleotides (pre-miRNA) is then produced, and its translocation into the cytoplasm is facilitated by RAN GTPase and Exportin 5 (XPO5). In the cytoplasm, pre-miRNAs are further processed by a protein complex that includes DICER1, TRBP, EIF2C1, EIF2C2, GEMIN3, and GEMIN4, resulting in the production of mature miRNAs (4). It has been predicted that there are more than 1,000 miRNA genes in the human genome (5), and $\sim 30 \%$ of human genes are regulated by miRNAs.

In the past few years, it was suggested that miRNAs in ALL are dysregulated. For example, in the study of Zhang et al., up to 171 miRNAs have been found to be differentially expressed between ALL patients and normal donors. These results suggest that dysregulation of these miRNAs may be associated with an increased risk for ALL. Changes in miRNAs function have the potential to affect the expression of a large number of genes, including genes involved in the origin and evolution of pediatric ALL $(6,7)$. Changes in miRNA function may occur through genetic variations (4). For example, single-nucleotide polymorphisms (SNPs) present in genes involved in miRNA processing can affect levels of miRNA expression, whereas SNPs in miRNA genes can affect miRNA biogenesis and function. There have been several polymorphisms found to be associated with other malignancies, and a recent pilot study has found an association between rs2910164 in mir-146a and ALL risk (8).

\footnotetext{
The first two authors contributed equally to this work.

'Department of Genetics, Physical Anthropology and Animal Physiology, University of the Basque Country (UPV/EHU), Leioa, Spain; ${ }^{2}$ Department of Hematology and Hemotherapy, University Hospital Cruces, Bilbao, Spain; ${ }^{3}$ Service of Pediatric Oncohematology, University Hospital La Paz, Madrid, Spain; ${ }^{4}$ Service of Pediatric Oncology and Hematology, University Hospital Vall d' Hebron, VHIR, Barcelona, Spain; ${ }^{5}$ Unit of Pediatric Oncohematology, University Hospital Miguel Servet, Zaragoza, Spain; ${ }^{6}$ Unit of Pediatric Oncohematology, University Hospital Donostia, San Sebastian, Spain; ${ }^{7}$ Unit of Pediatric Hematology/Oncology, University Hospital Cruces, Bilbao, Spain. Correspondence: Africa Garcia-Orad (africa.garciaorad@ehu.es)
} 


\section{Articles $\mid$ Gutierrez-Camino et al.}

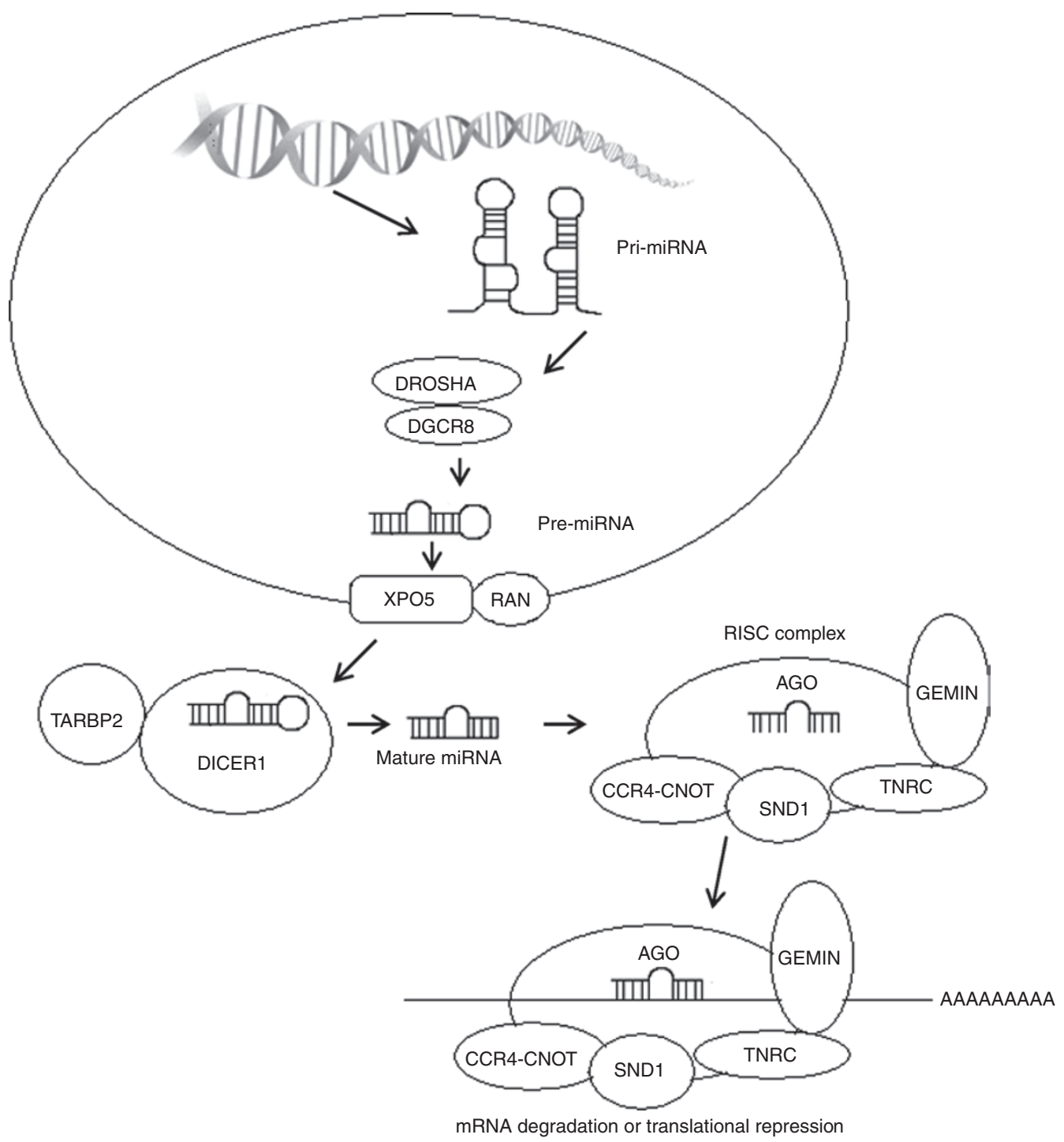

Figure 1. MicroRNA biogenesis pathway. Pre-miRNA, precursor microRNA; Pri-miRNA, primary double-stranded microRNA; RISC, RNA-induced silencing complex.

However, despite accumulating evidence that inherited genetic variation can contribute to a predisposition for pediatric ALL and the suggested role of miRNAs in the development of this disease, as well as the role of miRNA-related polymorphisms in cancer risk, the SNPs in miRNA genes and miRNA-processing genes have not been extensively studied in association with pediatric ALL risk.

Therefore, the aim of this study was to evaluate whether polymorphisms in pre-miRNAs, and/or miRNA-processing genes, contribute to a predisposition for childhood ALL.

\section{RESULTS}

\section{Genotyping Results}

A total of 213 patients with B-cell ALL (B-ALL) and 387 unrelated healthy controls were available for genotyping. Successful genotyping was achieved for 550 DNA samples (91.67\%). Of the SNPs, 106/118 (89.83\%) were genotyped satisfactorily. Failed genotyping was due to an absence of PCR amplification, insufficient intensity for cluster separation, poor cluster definition, or an inability to define clusters. The average genotyping rate for all SNPs was $98.12 \%$. Furthermore, of the 106 SNPs genotyped, 14 were not in Hardy-Weinberg equilibrium in the population of 387 healthy controls, and therefore, were not considered for further analysis. In total, 26 SNPs were excluded from the association study (see Supplementary Table S1 online), leaving 92 SNPs available for association studies.

\section{Analysis of Association}

To investigate if genetic variation influences the risk of ALL, the 92 polymorphisms successfully genotyped were compared between cases and controls. As shown in Tables 1 and 2, statistically significant associations $(P<0.05)$ were observed for 11 polymorphisms present in miRNA-related genes. Of these, three were located in pre-miRNAs (Table 1), and eight were located in miRNA-processing genes (Table 2).

Among the SNPs located in pre-miRNA genes, the SNP that was the most significantly associated with ALL risk was 
Table 1. Genotype frequencies of selected SNPs present in miRNA genes

\begin{tabular}{|c|c|c|c|c|c|c|c|c|}
\hline miRNA & SNP & $\begin{array}{l}\text { Best fitting } \\
\text { model }\end{array}$ & Genotype & Controls, $n(\%)$ & Cases, $n$ (\%) & OR $(95 \% \mathrm{Cl})$ & $P$ value & $P$ value $^{\mathrm{a}}$ \\
\hline \multirow[t]{2}{*}{ mir-612 } & rs12803915 & Additive & GG & $232(68)$ & $152(78)$ & Reference & 0.007 & 0.007 \\
\hline & & & $A A$ & $11(3)$ & $2(1)$ & & & \\
\hline mir-499 & rs3746444 & Additive & $A A$ & $206(59)$ & $138(69)$ & Reference & 0.009 & 0.01 \\
\hline \multirow[t]{2}{*}{ mir-449b } & rs10061133 & Dominant & $A A$ & $283(82)$ & $180(90)$ & Reference & 0.012 & 0.015 \\
\hline & & & GA/GG & $63(18)$ & $21(10)$ & $0.52(0.31-0.89)$ & & \\
\hline
\end{tabular}

$\mathrm{Cl}$, confidence interval; miRNA, microRNA; OR, odds ratio; SNP, single-nucleotide polymorphism.

a Calculated by multivariate logistic regressions to account for the possible confounding effect of sex.

Table 2. Genotype frequencies of selected SNPs present in miRNA-processing genes

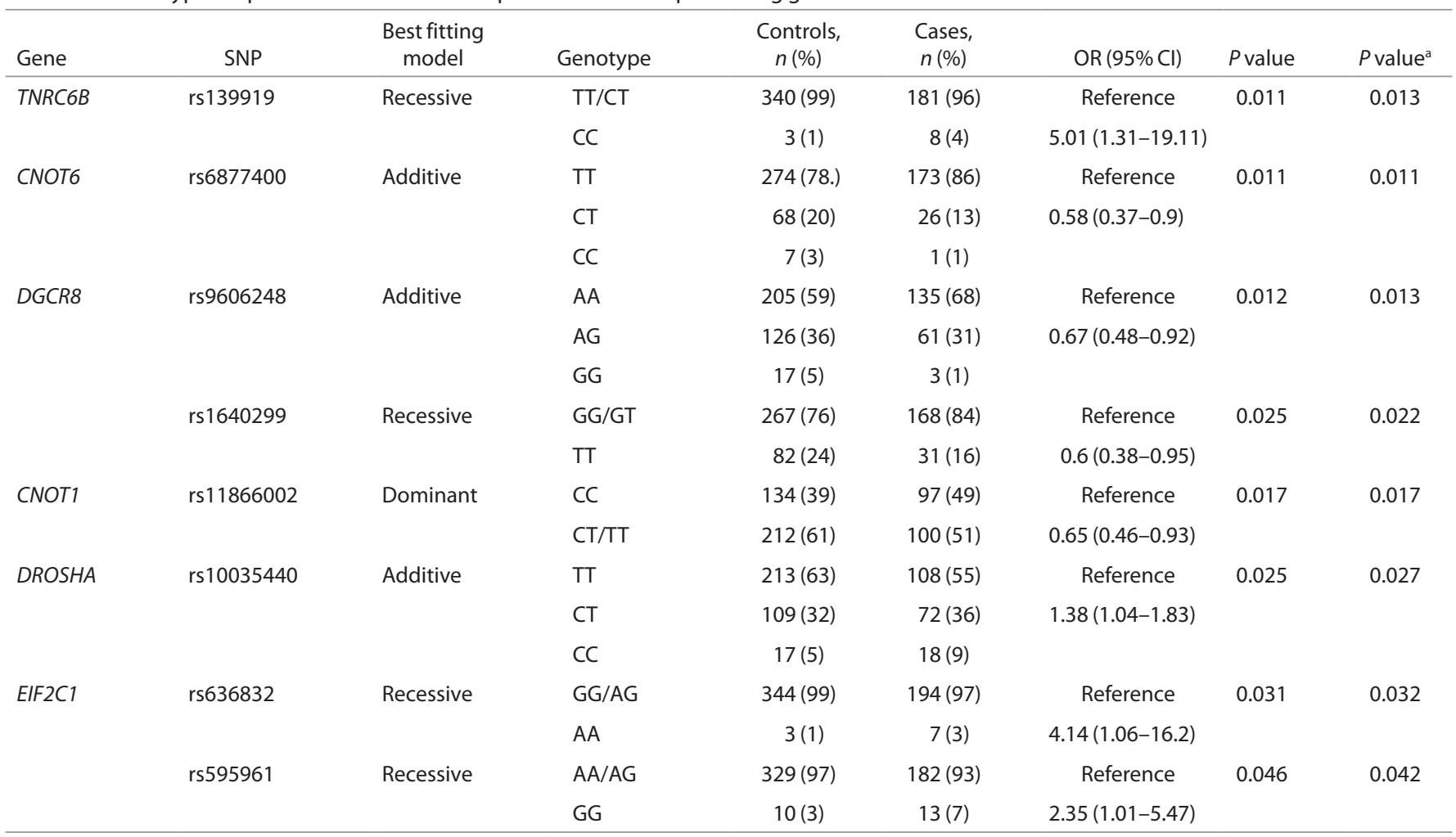

$\mathrm{Cl}$, confidence interval; miRNA, microRNA; OR, odds ratio; SNP, single-nucleotide polymorphism.

aCalculated by multivariate logistic regressions to account for the possible confounding effect of sex.

SNP rs12803915 in premature mir-612 (Figure 2a). The A allele for this SNP was found to be protective (odds ratio: 0.61 ; $95 \%$ confidence interval: $0.42-0.88 ; P=0.007$ ) in the $\log$-additive (GG vs. GA vs. AA) genetic model. The second most significant association involved SNP rs3746444 in mir-499 (Figure 2b). Moreover, this SNP is located in the seed region of mature miR-499-3p. The G allele of this SNP was found to be protective (odds ratio: $0.67 ; 95 \%$ confidence interval: $0.49-0.91 ; P=0.009$ ) in the log-additive (AA vs. AG vs. GG) genetic model. An association with rs10061133 in mir-449b was also identified (Table 1).
These two top SNPs, rs12803915 and rs3746444, were also studied in association with B-hyperdiploid ALL subtype (see Supplementary Tables S2 and S3 online) and chromosomal translocations (data not shown), and no significant differences were found.

In the genes of the miRNA-processing components, the most significant SNP was rs139919, a SNP located in the TNRC6B gene. In the recessive genetic model, the variant homozygous genotype, CC, of this SNP was associated with a 5.1-fold increase in ALL risk (95\% confidence interval: 1.31-19.11; $P=0.011$ ). Associations involving rs 9606248 and 


\section{Articles | Gutierrez-Camino et al.}
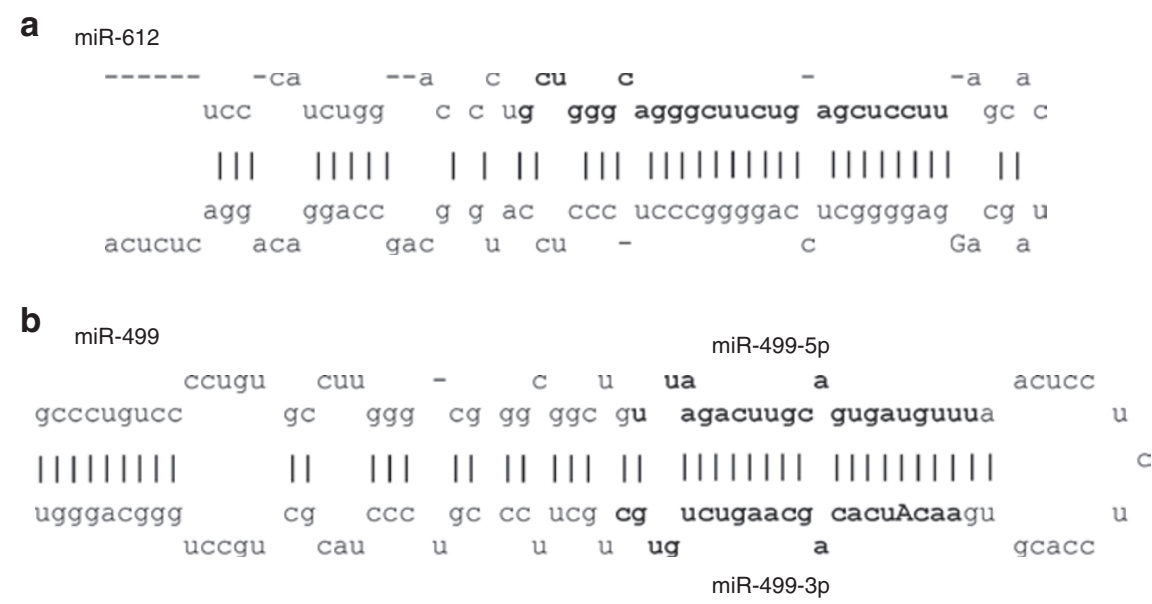

Figure 2. Schematic diagram of the hairpin loop structure of the mir-612 and mir-499. The sequence for mature microRNA is in bold. The polymorphisms sites are indicated in capital letters. (a) The $A>G$ polymorphism is located in the loop of mir-612; this variation might alter the secondary structure. (b) The $\mathrm{G}>\mathrm{A}$ polymorphism is located in the stem region opposite to the mature mir-499 sequence, which results in a change from A:U pair to G:U mismatch in the stem structure of mir-499 precursor.

rs1640299 in DGCR8, rs11866002 in CNOT1, rs6877400 in CNOT6, rs10035440 in DROSHA, and rs636832 and rs595961 in EIF2C1 were also identified (Table 2).

All the SNPs remained significantly associated with ALL risk after multivariate logistic regression to account for the possible confounding effect of sex. These SNPs did not reach the significant value when Bonferroni correction was applied.

\section{DISCUSSION}

In this study, 11 SNPs were found to be significantly associated with ALL susceptibility. These included three SNPs present in miRNA genes $(m i R-612, m i R-499$, and $m i R-449 b)$ and eight SNPs present in six miRNA biogenesis pathway genes (TNRC6B, DROSHA, DGCR8, EIF2C1, CNOT1, and CNOT6). Among them, rs12803915 in mir-612 and rs3746444 in mir499 exhibited a more significant association, with a $P$ value $<0.01$. In spite of not reaching a significant $P$ value after the restrictive Bonferroni correction, our results point to a putative role of these SNPs in ALL susceptibility, which could be of lower penetrance.

SNP rs12803915 located in the premature region of mir-612 showed the strongest association with ALL risk, with the A allele being protective $(P=0.007)$. To our knowledge, this is the first report of this SNP being associated with cancer risk. It has been suggested that a SNP in the premature region of a miRNA could alter its secondary structure and inhibit or enhance pri-miRNA processing (9). Therefore, SNPs in miRNAs could lead to dysregulation of miRNA expression (4). Accordingly, it has been shown that SNP rs12803915 significantly decreases mature miR612 levels, and this may represent a mechanism by which cancer risk is increased (10). One of the potential targets of mir-612 is IKZF2 (11), a member of the Ikaros family of zinc-finger proteins. This protein is a hematopoietic-specific transcription factor involved in the regulation of lymphocyte development, and other members of this family have been associated with ALL susceptibility $(2,3)$. Therefore, the presence of SNP rs12803915 in pre-mir-612 may contribute to an increased susceptibility to
ALL based on its capacity to affect the expression of mir-612, as well as its downstream targets, such as IKZF2.

The second most significant association identified in this study involved SNP rs3746444 $(P=0.009)$ located in premir-499. In this case, the $G$ allele was associated with a lower risk for ALL. This SNP has recently been implicated in the etiology of several types of cancer with controversial results. The results of this study are consistent with those of three previous studies, in which the rs3746444 GG genotype was shown to be associated with a decreased risk of cancer (12-14). By contrast, in six other studies $(5,15-19)$, the GG genotype was found to be associated with a higher risk of cancer development. There have also been studies that have found no association between this SNP and cancer risk $(8,20-24)$. The inconsistency of these results may be due to differences in the carcinogenic mechanisms of different cancers, as well as differences in genetic backgrounds (25). Correspondingly, a meta-analysis observed that a tendency for reduced cancer risk was associated with the mir499 rs3746444 GG genotype in Caucasian populations (26).

This SNP, rs3746444, is located in pre-mir-499, in the premature sequences of mir-499-5p and mir-499-3p and in the seed sequence of mir-499-3p. Therefore, it could have a double effect. On the one hand, a SNP located in a pre-miRNA region has the potential to impact the processing of pre-miRNAs into mature miRNAs (14). Correspondingly, different genotypes of rs3746444 in pre-mir-499 have been associated with dysregulated expression of mir-499-5p in a colorectal cancer model (5). These data are of particular interest considering that mir-499-5p seems to be upregulated in ALL (6). On the other hand, SNP rs3746444 is also present in the seed region of a mature miRNA-499-3p and could be essential for the accurate recognition of target mRNA sequences. Potential targets of mir-499-3p (11) include FOXO1A (a transcription factor that is dysregulated in B-ALL (27)), MS4A1 or CD20 (a B-lymphocyte surface molecule that plays a role in the development and differentiation of $B$ cells into plasma cells), and PBX1 (pre-B-cell leukemia homeobox 1 that is a dysregulated 
transcription factor in ALL) (28). Therefore, alterations in the sequence of mir-499-5p and mir-499-3p, which affect expression of these miRNAs and/or the binding of mir-499-3p to target mRNAs, may have functional consequences for ALL.

Among the SNPs located in pre-miRNA genes, other interesting result was that the rs $10061133 \mathrm{G}$ allele in mir- $449 b$ was associated with a decreased risk of ALL $(P=0.012)$. To our knowledge, this is the first time that this polymorphism has been associated with cancer risk. Based on its location, this polymorphism is also present in the mature sequence of the miRNA; therefore, it could affect the strength of miRNAmRNA binding, as well as miRNA levels. This miRNA has also been found to be upregulated in endometrial cancer and bladder cancer $(29,30)$.

However, in this larger population, we have not replicated the association previously found in a small pilot study between rs2910164 in mir-146a and ALL risk (8).

When we analyzed 72 polymorphisms present in miRNA biogenesis pathway, 8 of these were found to be significantly associated with ALL risk. Polymorphism rs139919 in TNRC6B was the SNP most highly associated with ALL susceptibility among the miRNA-processing genes, with the CC genotype associated with an increase in ALL risk $(P=0.011)$. TNRC6B encodes an RNA interference machinery component, which contributes to the RNA-induced silencing complex, and is crucial for miRNA-dependent translational repression or degradation of target mRNAs (31). Although this SNP has not been analyzed previously, other genetic variants in TNRC6B have been associated with prostate cancer risk $(31,32)$. It has also

Table 3 Characteristics of the B-ALL patients and controls examined in this study

\begin{tabular}{|c|c|c|}
\hline & Patients & Controls \\
\hline No. of individuals ( $n$ ) & 213 & 387 \\
\hline Male, $n(\%)$ & $124(56)$ & $199(51)$ \\
\hline Female, $n(\%)$ & $95(43)$ & $187(48)$ \\
\hline Mean age $\pm S E, y$ & $5.7 \pm 3.5$ & $51.2 \pm 7.7$ \\
\hline \multicolumn{3}{|l|}{ Age at diagnosis } \\
\hline $1-9 y$ & $173(81)$ & - \\
\hline$\geq 10 y$ & $38(18)$ & - \\
\hline \multicolumn{3}{|c|}{ Leukocytes at diagnosis (WBC) } \\
\hline$<20\left(\times 10^{9} / \mathrm{l}\right)$ & $94(30)$ & - \\
\hline $20-200\left(\times 10^{9} / \mathrm{I}\right)$ & $54(25)$ & - \\
\hline$>200\left(\times 10^{9} / \mathrm{I}\right)$ & $6(3)$ & - \\
\hline Hyperdiploid, $n$ (\%) & $55(26)$ & - \\
\hline No hyperdiploid, $n$ (\%) & $107(50)$ & - \\
\hline \multicolumn{3}{|c|}{ Chromosomal translocations } \\
\hline ETV6-RUNX1 & $28(13)$ & - \\
\hline$M L L$ rearranged & $10(5)$ & - \\
\hline$B C R-A B L$ & $5(2)$ & - \\
\hline$E 2 A-P B X 1$ & $3(1)$ & - \\
\hline
\end{tabular}

B-ALL, B-cell acute lymphoblastic leukemia; WBC, white blood cell. been suggested that alterations in the expression of TNRC6B are due to genetic variations that may affect mRNA levels that are normally regulated by $T N R C 6 B$, thereby affecting carcinogenesis (32).

We have also found other SNPs located in genes which produce proteins that contribute to the RNA-induced silencing complex associated with ALL susceptibility. These included two SNPs in EIF2C1 (rs595961 and rs636832), one SNP in CNOT1 (rs11866002), and one SNP in CNOT6 (rs6877400). The SNPs rs595961 and rs636832 in EIF2C1 have been previously shown to be associated with renal cell carcinoma risk in males (33) and lung cancer (34), respectively. However, to our knowledge, this is the first study to identify an association between the SNPs, rs11866002 in CNOT 1 and rs6877400 in CNOT6, and cancer risk. In addition, it is hypothesized that rs595961, rs11866002, and rs6877400 have putative roles in transcription and/or regulation of splicing events, thereby affecting gene expression. SNPs that affect expression levels of these proteins may have deleterious effects on miRNA-mRNA interactions and may affect cancer development and progression. Correspondingly, EIF2C1 is frequently lost in human cancers such as Wilms tumor, neuroblastoma, and carcinomas of the breast, liver, and colon (35).

Among the genes of biogenesis machinery, when we studied genes that contribute to the processing of pri-miRNAs to pre-miRNAs, we identified an association between SNPs and ALL risk. These SNPs included rs 10035440 in DROSHA, and rs9606248 and rs1640299 in DGCR8. Interestingly, these three SNPs have putative roles in transcriptional regulation and may affect the expression levels of DROSHA or DGCR8. As a result, levels of miRNAs could be affected, leading to an increased ALL risk. Consistent with this hypothesis, a differentially expressed miRNA profile and DROSHA gene expression have been observed in relation to another SNP, rs640831, being present in the lung tissue (36). Furthermore, increased expression of DROSHA and DGCR8 has been shown to dysregulate miRNAs present in the pleomorphic adenomas of the salivary gland (37). In contrast, decreased expression of DROSHA and DGCR8 have been shown to accelerate cellular transformation and tumorigenesis (38). Surprisingly, the association of these polymorphisms with the risk to develop other tumors has previously been analyzed $(25,33,39)$, and no significant association was found. However, they had not been analyzed in ALL patients until now.

In conclusion, the results of this study indicate that SNP rs12803915 located in pre-mir-612 and SNP rs3746444 located in pre-mir-499 may represent novel markers of B-ALL susceptibility. It would be of great interest to confirm these results in different cohorts of patients. To our knowledge, this is the first extensive study to report miRNA-related SNPs associated with ALL risk.

\section{METHODS}

\section{Study Participants}

A total of 213 children (1-15 y) of European origin all diagnosed with precursor B-ALL in the Pediatric Oncology Units of five Spanish hospitals (Hospital Cruces, Hospital Donostia, Hospital 
Vall d'Hebrón, Hospital La Paz, and Hospital Miguel Servet) were enrolled in this study. These patients were the entire incident population diagnosed and treated in the participating centers between 1995 and 2011. In addition, 387 Spanish healthy individuals of European origin with no previous history of cancer from the collection C.0001171 registered in the Institute of Health Carlos III were enrolled as controls (Table 3). Patients were classified with B-hyperdiploid ALL if the DNA index was > 1.16 and/or the karyotype had more than 50 chromosomes. For 51 cases out of 213 B-ALL patients, cytogenetic data were not available. Informed consent was obtained from all participants, or from their parents, prior to sample collection. The study was approved by the Ethics Committee for Clinical Research and conducted in accordance with the Declaration of Helsinki.

\section{Selection of Genes and Polymorphisms}

Twenty-one genes involved in miRNA biogenesis and processing, as determined based on available literature and the Patrocles (http:// www.patrocles.org/; University of Liege, Liège, Belgium) database, were selected for the analysis. For each gene, all of the SNPs with potential functional effects were examined using F-SNP (http:// compbio.cs.queensu.ca/F-SNP/; Queen's University, Kingston, Canada), Fast-SNP (http://fastsnp.ibms.sinica.edu.tw; Academia Sinica, Taipei, Taiwan), polymirTS (http://compbio.uthsc.edu/ miRSNP/; University of Tennessee Health Science Center, Memphis, TN), and Patrocles databases. Functional effects were considered to be those that resulted in amino acid changes and/or alternate splicing, those that were located in the promoter region of putative transcription factor-binding sites, or those that disrupted or created miRNAs binding interactions. SNPs previously included in association studies were also examined. The final selection of SNPs was made based on those having a minor allele frequency greater than $5 \%$ (i.e., $\geq 0.05$ ) in European/Caucasian populations.

Considering that miRNAs can regulate a wide range of genes and that the number of polymorphisms in miRNAs was affordable, we selected all the known SNPs at the moment of the selection with a minor allele frequency $>0.01$ in European/Caucasoid populations, using Patrocles and Ensembl (http://www.ensembl.org/; Welcome Trust Genome Campus, Cambridge, UK) databases and literature review.

\section{Genotyping}

Genomic DNA was extracted from remission (containing less than $5 \%$ blast cells) peripheral blood, bone marrow slides or granulocytes isolated with Ficoll-Plaque PLUS (GE Healthcare Life Sciences, Piscataway, NJ), using the phenol-chloroform method (40) or from saliva samples using Oragene DNA kit (DNA Genotek, Ottawa, Canada) according to the manufacturer's instructions.

SNP genotyping was performed using TaqMan OpenArray Genotyping technology (Applied Biosystems, Carlsbad, CA) according to the published Applied Biosystems protocol. Initially, 131 SNPs were considered for analysis. After considering compatibility with the Taqman OpenArray platform, 118 SNPs were included in a Taqman OpenArray Plate (Applied Biosystems), and these included 72 SNPs present in 21 genes involved in miRNA biogenesis and 46 SNPs present in 42 pre-miRNA genes (see Supplementary Tables S4 and S5 online).

Data were analyzed using Taqman Genotyper software (Applied Biosystems) for genotype clustering and genotype calling. Duplicate samples were genotyped across the plates.

\section{Statistical Analyses}

Statistical analyses were performed using R software (version v2.14.1; Institute for Statistics and Mathematics, Wien, Austria). To identify any deviation in Hardy-Weinberg equilibrium for the healthy controls $(n=387)$, a $\chi^{2}$ test was used. The association between genetic polymorphisms in B-ALL patients and controls was also evaluated using the $\chi^{2}$ or Fisher's exact test. Fisher's exact test was used if a genotype class had less than five individuals. We also tested the association considering genetic characteristics (hyperdiploid subtype and chromosomal translocations). The effect sizes of the associations were estimated by the odds ratio from univariate logistic regression and multivariate logistic regression to account for the possible confounding effect of sex.

The most significant test among codominant (major allele homozygotes vs. heterozygotes and major allele homozygotes vs. minor allele homozygotes), dominant (major allele homozygotes vs. heterozygotes + minor allele homozygotes), recessive (major allele homozygotes + heterozygotes vs. minor allele homozygotes), and additive (dosesdependent effect: major allele homozygotes vs. heterozygotes vs. minor allele homozygotes) genetic models was selected. In all cases, the significance level was set at $5 \%$. The results were adjusted for multiple comparisons using the Bonferroni correction.

\section{SUPPLEMENTARY MATERIAL}

Supplementary material is linked to the online version of the paper at http:// www.nature.com/pr

\section{STATEMENT OF FINANCIAL SUPPORT}

A.G.-C. was supported by a predoctoral grant from the Gangoiti Barrera Foundation, Bilbao, Spain. E.L.-L. was supported by a predoctoral grant of the Basque Government and "Fellowship for recent doctors until their integration in postdoctoral programs" by the Investigation Vice-rector's office of the University of Basque Country (UPV/EHU). This project was supported by Spanish Thematic Network of Cooperative Research in Cancer (RD/06/0020/0048), the Basque Government (IT661-13, S-PE12UN060), and UPV/EHU (UFI11/35). Support by SGlker (UPV/EHU) is gratefully acknowledged.

Disclosure: The authors have no conflicts of interest to disclose.

\section{REFERENCES}

1. Ellinghaus E, Stanulla M, Richter G, et al. Identification of germline susceptibility loci in ETV6-RUNX1-rearranged childhood acute lymphoblastic leukemia. Leukemia 2012;26:902-9.

2. Treviño LR, Yang W, French D, et al. Germline genomic variants associated with childhood acute lymphoblastic leukemia. Nat Genet 2009;41:1001-5.

3. Papaemmanuil E, Hosking FJ, Vijayakrishnan J, et al. Loci on 7p12.2, $10 \mathrm{q} 21.2$ and $14 \mathrm{q} 11.2$ are associated with risk of childhood acute lymphoblastic leukemia. Nat Genet 2009;41:1006-10.

4. Ryan BM, Robles AI, Harris CC. Genetic variation in microRNA networks: the implications for cancer research. Nat Rev Cancer 2010;10:389-402.

5. Vinci S, Gelmini S, Mancini I, et al. Genetic and epigenetic factors in regulation of microRNA in colorectal cancers. Methods 2013;59:138-46.

6. Zhang H, Yang JH, Zheng YS, et al. Genome-wide analysis of small RNA and novel microRNA discovery in human acute lymphoblastic leukemia based on extensive sequencing approach. PLoS One 2009;4:e6849.

7. Schotte D, Akbari Moqadam F, Lange-Turenhout EA, et al. Discovery of new microRNAs by small RNAome deep sequencing in childhood acute lymphoblastic leukemia. Leukemia 2011;25:1389-99.

8. Hasani SS, Hashemi M, Eskandari-Nasab E, Naderi M, Omrani M, Sheybani-Nasab M. A functional polymorphism in the miR-146a gene is associated with the risk of childhood acute lymphoblastic leukemia: a preliminary report. Tumour Biol 2014;35:219-25.

9. Salzman DW, Weidhaas JB. SNPing cancer in the bud: microRNA and microRNA-target site polymorphisms as diagnostic and prognostic biomarkers in cancer. Pharmacol Ther 2013;137:55-63.

10. Kim HK, Prokunina-Olsson L, Chanock SJ. Common genetic variants in miR-1206 (8q24.2) and miR-612 (11q13.3) affect biogenesis of mature miRNA forms. PLoS One 2012;7:e47454.

11. Friedman RC, Farh KK, Burge CB, Bartel DP. Most mammalian mRNAs are conserved targets of microRNAs. Genome Res 2009;19:92-105.

12. Kim WH, Min KT, Jeon YJ, et al. Association study of microRNA polymorphisms with hepatocellular carcinoma in Korean population. Gene 2012;504:92-7.

13. Liu Z, Li G, Wei S, et al. Genetic variants in selected pre-microRNA genes and the risk of squamous cell carcinoma of the head and neck. Cancer 2010;116:4753-60.

14. Ahn DH, Rah H, Choi YK, et al. Association of the miR-146aC>G, miR$149 \mathrm{~T}>\mathrm{C}$, miR-196a2T $>\mathrm{C}$, and miR-499A $>\mathrm{G}$ polymorphisms with gastric cancer risk and survival in the Korean population. Mol Carcinog 2013;52:Suppl 1:E39-51. 
15. Xiang Y, Fan S, Cao J, Huang S, Zhang LP. Association of the microRNA-499 variants with susceptibility to hepatocellular carcinoma in a Chinese population. Mol Biol Rep 2012;39:7019-23.

16. Zhou B, Wang K, Wang Y, et al. Common genetic polymorphisms in premicroRNAs and risk of cervical squamous cell carcinoma. Mol Carcinog 2011;50:499-505.

17. Hu Z, Chen J, Tian T, et al. Genetic variants of miRNA sequences and nonsmall cell lung cancer survival. J Clin Invest 2008;118:2600-8.

18. Alshatwi AA, Shafi G, Hasan TN, et al. Differential expression profile and genetic variants of microRNAs sequences in breast cancer patients. PLoS One 2012;7:e30049.

19. George GP, Gangwar R, Mandal RK, Sankhwar SN, Mittal RD. Genetic variation in microRNA genes and prostate cancer risk in North Indian population. Mol Biol Rep 2011;38:1609-15.

20. Akkiz H, Bayram S, Bekar A, Akgöllü E, Üsküdar O. Genetic variation in the microRNA-499 gene and hepatocellular carcinoma risk in a Turkish population: lack of any association in a case-control study. Asian Pac J Cancer Prev 2011;12:3107-12.

21. Catucci I, Yang R, Verderio P, et al. Evaluation of SNPs in miR-146a, miR196a2 and miR-499 as low-penetrance alleles in German and Italian familial breast cancer cases. Hum Mutat 2010;31:E1052-7.

22. Min KT, Kim JW, Jeon YJ, et al. Association of the miR-146aC $>$ G, $149 \mathrm{C}>\mathrm{T}$, $196 \mathrm{a} 2 \mathrm{C}>\mathrm{T}$, and $499 \mathrm{~A}>\mathrm{G}$ polymorphisms with colorectal cancer in the Korean population. Mol Carcinog 2012;51:Suppl 1:E65-73.

23. Okubo M, Tahara T, Shibata T, et al. Association between common genetic variants in pre-microRNAs and gastric cancer risk in Japanese population. Helicobacter 2010;15:524-31.

24. Tian T, Shu Y, Chen J, et al. A functional genetic variant in microRNA$196 \mathrm{a} 2$ is associated with increased susceptibility of lung cancer in Chinese. Cancer Epidemiol Biomarkers Prev 2009;18:1183-7.

25. Yang H, Dinney CP, Ye Y, Zhu Y, Grossman HB, Wu X. Evaluation of genetic variants in microRNA-related genes and risk of bladder cancer. Cancer Res 2008;68:2530-7.

26. Qiu MT, Hu JW, Ding XX, et al. Hsa-miR-499 rs3746444 polymorphism contributes to cancer risk: a meta-analysis of 12 studies. PLoS One 2012;7:e50887.

27. Andersson A, Ritz C, Lindgren D, et al. Microarray-based classification of a consecutive series of 121 childhood acute leukemias: prediction of leukemic and genetic subtype as well as of minimal residual disease status. Leukemia 2007;21:1198-203.

28. LiZ, Zhang W, Wu M, et al. Gene expression-based classification and regulatory networks of pediatric acute lymphoblastic leukemia. Blood 2009;114:4486-93.

29. Chung TK, Lau TS, Cheung TH, et al. Dysregulation of microRNA-204 mediates migration and invasion of endometrial cancer by regulating FOXC1. Int J Cancer 2012;130:1036-45.

30. Catto JW, Miah S, Owen HC, et al. Distinct microRNA alterations characterize high- and low-grade bladder cancer. Cancer Res 2009;69:8472-81.

31. Tao S, Wang Z, Feng J, et al. A genome-wide search for loci interacting with known prostate cancer risk-associated genetic variants. Carcinogenesis 2012;33:598-603.

32. Sun J, Zheng SL, Wiklund F, et al. Sequence variants at 22q13 are associated with prostate cancer risk. Cancer Res 2009;69:10-5.

33. Horikawa Y, Wood CG, Yang H, et al. Single nucleotide polymorphisms of microRNA machinery genes modify the risk of renal cell carcinoma. Clin Cancer Res 2008;14:7956-62.

34. Kim JS, Choi YY, Jin G, et al. Association of a common AGO1 variant with lung cancer risk: a two-stage case-control study. Mol Carcinog 2010;49:913-21.

35. Koesters R, Adams V, Betts D, et al. Human eukaryotic initiation factor EIF2C1 gene: cDNA sequence, genomic organization, localization to chromosomal bands 1p34-p35, and expression. Genomics 1999;61:210-8.

36. Rotunno M, Zhao Y, Bergen AW, et al. Inherited polymorphisms in the RNA-mediated interference machinery affect microRNA expression and lung cancer survival. Br J Cancer 2010;103:1870-4.

37. Zhang X, Cairns M, Rose B, et al. Alterations in miRNA processing and expression in pleomorphic adenomas of the salivary gland. Int J Cancer 2009;124:2855-63.

38. Kumar MS, Lu J, Mercer KL, Golub TR, Jacks T. Impaired microRNA processing enhances cellular transformation and tumorigenesis. Nat Genet 2007;39:673-7.

39. Sung H, Lee KM, Choi JY, et al. Common genetic polymorphisms of microRNA biogenesis pathway genes and risk of breast cancer: a casecontrol study in Korea. Breast Cancer Res Treat 2011;130:939-51.

40. Sambrook J, Russell DW. Preparation and Analysis of Eukaryotic Genomic DNA. Molecular Cloning: A Laboratory Manual. 3rd edn. Cold Spring Harbor, USA: CSHL Press, 2001:4-12. 